\title{
Francisolã
}

\section{LES PROBLÈMES DE PRONONCIATION CHEZ LES LYCÉENS DE FRANÇAIS LANGUE ÉTRANGÈRE DANS LE SUD DE LA THAÏLANDE}

\author{
Sirima PURINTHRAPIBAL \\ Université Prince de Songkla, campus de Pattani, Thaïlande \\ spurinthrapibal@hotmail.com \\ Article reçu le 5 avril 2016 | révisé depuis le 5 mai 2016 | accepté le 20 juin 2016
}

\begin{abstract}
RÉSUMÉ. Les différences phonologiques dans les langues thaïe et française impliquent des difficultés de prononciation aux apprenants thaïlandais de FLE. Cette recherche vise à étudier leurs problèmes de prononciation du français. Elle est menée auprès de 30 lycéens de FLE. Le test de prononciation d'une liste de 65 mots et de 45 paires minimales constitue le matériel d'expérimentation. La recherche nous met en évidence que certains problèmes phonétiques rencontrés par ces lycéens sont issus de l'influence de leur langue maternelle, d'autres de l'influence de leur première langue étrangère apprise, l'anglais. En outre, la complexité des systèmes phonétiques du français est aussi une autre cause de problèmes de prononciation chez ces apprenants.
\end{abstract}

Mots-clés: apprenants thaillandais, français langue étrangère, interférence phonique, problèmes, prononciation.

\begin{abstract}
Phonological differences between Thai and French languages involve pronunciation difficulties for Thai learners of French as a foreign language. This research aims to study French pronunciation problems of Thai learners. It is conducted with 30 secondary school students of French. Pronunciation test consisting of a list of 60 words and 45 minimal pairs is the experimental equipment. The result of this research shows that phonetic problems faced by these students are caused by the influence of mother tongue and their first foreign language learned, English. Furthermore, the complexity of the French phonetics systems is also another cause of their pronunciation problems.
\end{abstract}

Keywords: french as a foreign language, phonics interference, problems, pronunciation, Thai learners.

\section{INTRODUCTION}

Le thaï et le français viennent de familles linguistiques différentes. Cette différence d'origine engendre toute une série de caractéristiques distinctes tant au niveau $\mathrm{du}$ système phonologique et morphosyntaxique que du système sémantico-pragmatique. Il va de soi que de ces différences proviennent les nombreuses difficultés rencontrées par les étudiants thaïlandais apprenant le français langue étrangère, en particulier celles relatives à la prononciation. De plus, des études de recherche menées auprès des apprenants de français en Thaïlande (Boonprom, 2009a, 2009b ; Purinthrapibal, 2008 ; Roungtheera, 2011 ; Ngammana, 2011) ont révélé que des difficultés de prononciation $\mathrm{du}$ français 
rencontrés par ces apprenants relèvent également de l'influence de leur langue 1 et de l'anglais, leur première langue étrangère. En principe, les apprenants cherchent à mettre en parallèle des éléments linguistiques dans la langue source ou la première langue étrangère qui sont proches de ceux de la langue cible. Ensuite, ils créent un transfert négatif en langue cible des habitudes articulatoire en langue source ou l'interférence phonique. Celle-ci manifeste donc sous forme de fautes de prononciation qui peuvent être soit inhérentes au système de la langue cible soit spécifiques. Les problèmes de prononciation constituent donc la question principale mise en cause pour notre étude de recherche qui a pour but d'étudier non seulement les problèmes de prononciation $\mathrm{du}$ français et les interférences phonologiques dans la prononciation du français chez les apprenants thaïlandais, mais aussi les causes de ces problèmes.

Dans la didactique des langues étrangères, l'analyse contrastive occupe un rôle important. Elle s'attache à établir les conditions qui limitent dans la langue cible l'emploi de telle ou telle forme linguistique. On ne retient en analyse contrastive que les cas qui posent problème, qui représentent des points de divergence pouvant entraîner des erreurs (Jamet, 2000). La notion d'interférence est centrale dans l'analyse contrastive. En effet, les études contrastives des systèmes des langues permettent d'identifier les similitudes et les contrastes significatifs qui pourraient exister entre deux langues. Les différences entre deux langues créent des difficultés aux apprenants puisqu'elles sont sources d'interférence. L'analyse contrastive contribue donc à prédire des zones d'interférentielles permettant de prévenir les fautes et dans un second temps d'y remédier (Cuq, 2003).

\section{MÉTHODE}

Notre recherche se distingue en deux phases :

1. La première phase relève de l'analyse comparative des caractéristiques phonologiques du thaï par rapport à celles $\mathrm{du}$ français.

2. La seconde phase concerne une étude expérimentale pour examiner les problèmes de prononciation de français. Elle est menée auprès de 30 lycéens de Mathayomsuksa 6 qui apprennent le français langue étrangère depuis deux ans ou environs 350 heures de trois écoles secondaires dans le Sud de la Thaillande pendant le premier semestre de l'année scolaire 2012 (du début juin à la fin septembre 2012). Ces sujets sont appelés l'un après l'autre à faire le test de prononciation composé d'une liste de 65 mots et de 45 paires minimales (en annexe). Et les échantillons sont enregistrés par le logiciel informatique Adobe Audition pour l'analyse des résultats.

\section{RÉSULTATS ET DISCUSSION}

\section{L'analyse comparative}

L'analyse comparative des caractéristiques phonologiques du thaï par rapport à celles du français nous permet de constater un certain nombre de ressemblances et de différences entre ces deux langues comme montrent les tableaux ci-dessous.

Tableau 1. Les phonèmes consonantiques du thaï selon les points et les modes d'articulation

\begin{tabular}{|c|c|c|c|c|c|c|c|}
\hline \multicolumn{2}{|r|}{ Mode d'articulation } & \multicolumn{6}{|c|}{ Point d'articulation } \\
\hline & & $\frac{\mathscr{0}}{\frac{\pi}{0}}$ & 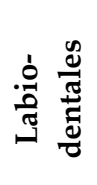 & 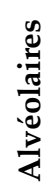 & $\frac{\infty}{\underset{J}{*}}$ & $\frac{d}{2}$ & 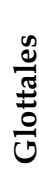 \\
\hline \multirow{3}{*}{$\frac{\sum_{0}^{\infty}}{0}$} & sourdes aspirées & $\mathrm{p}^{\mathrm{h}}$ & & $t^{\text {h }}$ & $\mathrm{t} 6^{\mathrm{h}}$ & $\mathrm{k}^{\mathrm{h}}$ & \\
\hline & sourdes non aspirées & $\mathrm{p}$ & & $\mathrm{t}$ & t6 & $\mathrm{k}$ & $?$ \\
\hline & sonores & $b$ & & $\mathrm{~d}$ & & & \\
\hline
\end{tabular}




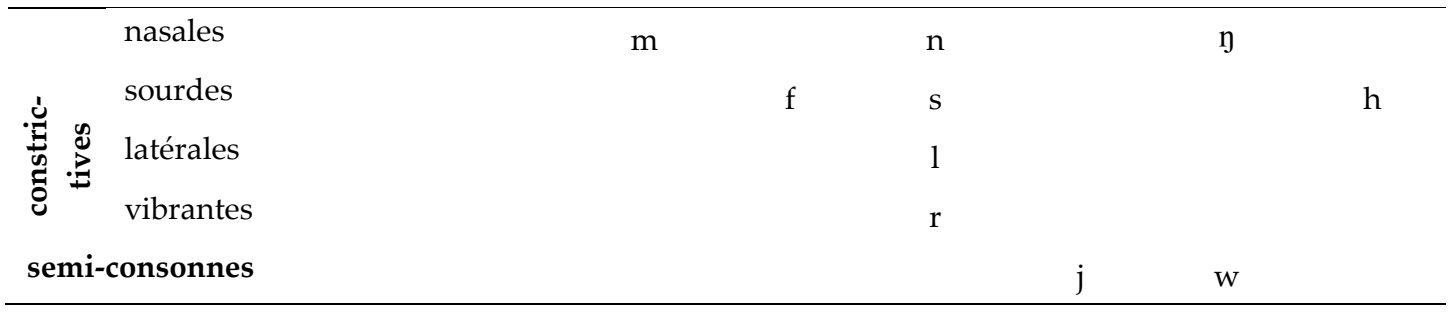

(Ninlagarn, 1993, p.7)

Tableau 2. Les phonèmes consonantiques du français selon les points et les modes d'articulation

Mode d'articulation

Point d'articulation

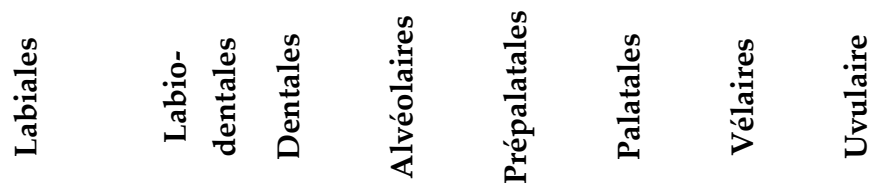

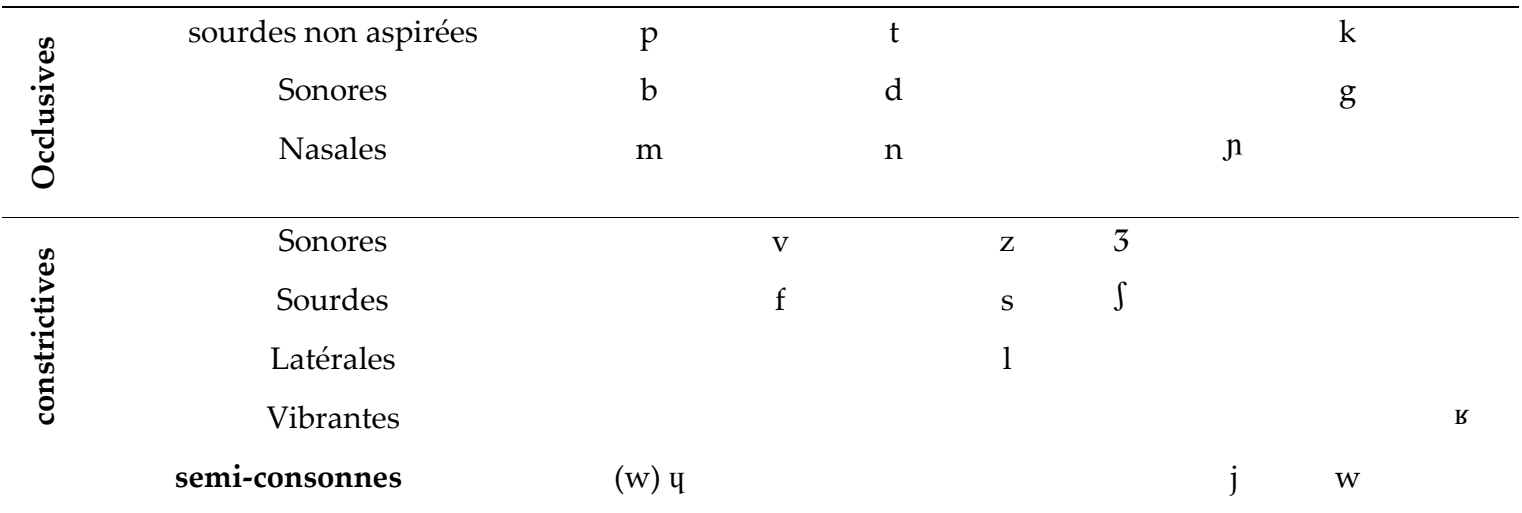

(Léon, 1992, p.82)

Ces tableaux nous montrent que le nombre de phonèmes consonantiques est le même dans les deux langues. La langue thaïe comprend 21 phonèmes consonantiques dont 2 semi-consonnes alors que le français dispose aussi de 21 phonèmes dont 3 semiconsonnes (Wioland, 1991). Mais au niveau de la prononciation, les réalisations dans les deux langues sont différentes.

Certaines unités phonémiques des consonnes et semi-consonnes se ressemblent dans les deux langues:/p/, /t/, /k/, /b/, /d/, $/ \mathrm{m} /, / \mathrm{n} /, / \mathrm{f} /, \mathrm{s} /, \mathrm{l} / \mathrm{l} / \mathrm{j} /$ et $/ \mathrm{w} /$.

Les deux systèmes sont pourtant différents. Certaines consonnes existent en français mais non en thaï telles que :

- les constrictives sonores: /v/, /z/, /3/ et la constrictive sourde $/ \mathrm{s} /$

- l'occlusive vélaire sonore /g/

- la vibrante uvulaire /ь/,

- la semi-consonne /4/.
A l'inverse, nous constatons que plusieurs consonnes sont utilisées en thaï mais ne connaissent pas d'équivalent en français :

- les articulations affriquées sourde /tc/, et sourde aspirée $/ \mathrm{t} \varphi^{\mathrm{h}} /$,

- les occlusives bilabiale sourde aspirée $/ \mathrm{p}^{\mathrm{h}} /$, alvéolaire sourde aspirée $/ \mathrm{t}^{\mathrm{h}} /$ et palatovélaire sourde aspirée $/ \mathrm{k}^{\mathrm{h}} /$,

- 1'occlusive vélaire sonore nasale /n/,

- l'occlusive glottale sourde non aspirée / ?/

- la vibrante alvéolaire /r/,

- la consonne glottale $/ \mathrm{h} /$.

Les semi-consonnes $/ \mathrm{j} /$ et $/ \mathrm{w} /$ en thaï fonctionnent comme en français. Elles remplissent une fonction de consonnes soit initiales soit finales et forment donc, comme toute autre consonne, une syllabe quand elles sont combinées avec une voyelle.

De plus, en comparant le système vocalique des deux langues thaïe et française, 
nous trouvons que leurs phonèmes les tableaux suivants. vocaliques sont différents comme montrent

Tableau 3. Les phonèmes vocaliques en thaï en fonction de la zone d'articulation et du degré d'aperture

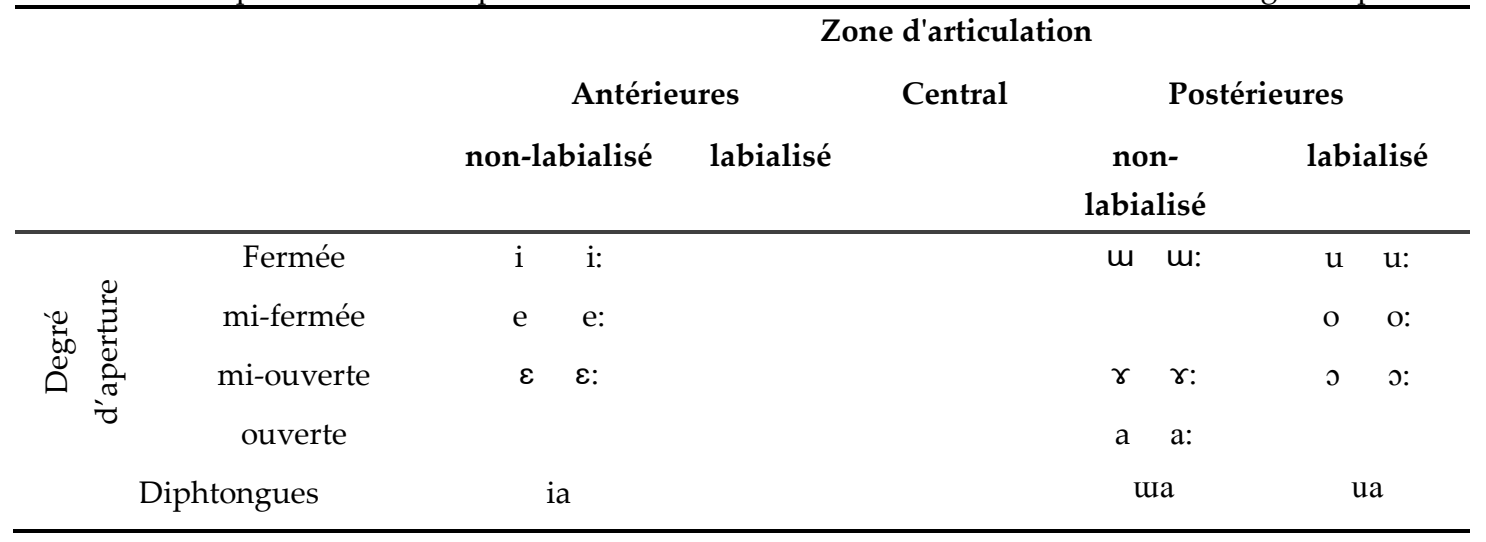

(Boonprome, 2009a, p.12)

Tableau 4. Les phonèmes vocaliques du français en fonction de la zone d'articulation et du degré d'aperture

\begin{tabular}{|c|c|c|c|c|c|c|c|}
\hline & & & \multicolumn{5}{|c|}{ Zone d'articulation } \\
\hline & & & \multicolumn{2}{|c|}{ Antérieures } & \multirow[t]{2}{*}{ Central } & \multicolumn{2}{|c|}{ Postérieures } \\
\hline & & & $\begin{array}{c}\text { non- } \\
\text { labialise }\end{array}$ & Labialisé & & $\begin{array}{c}\text { non- } \\
\text { labialisé }\end{array}$ & labialisé \\
\hline \multirow{4}{*}{ 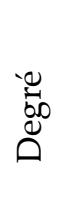 } & \multirow{4}{*}{ 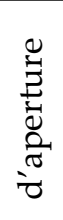 } & Fermée & $\mathrm{i}$ & $\mathrm{Y}$ & & & $\mathrm{u}$ \\
\hline & & mi-fermée & e & $\varepsilon$ & $\partial$ & & $\mathrm{O}$ \\
\hline & & mi-ouverte & $\varepsilon \quad \tilde{\varepsilon}$ & $œ \quad œ \tilde{~}$ & & & $0 \quad \tilde{}$ \\
\hline & & Ouverte & $\mathrm{a}$ & & & & $a$ \\
\hline
\end{tabular}

(Léon, 1992, p.82)

Selon les tableaux ci-dessus, le thaï possède dix-huit unités phonémiques vocaliques simples. Elles se distinguent en neuf phonèmes brefs et neuf phonèmes longs correspondants, à l'exception des deux «A » /a/ et /a:/ dont l'opposition d'articulation est concrète: l'un est antérieur, l'autre est postérieur. A ces dix-huit phonèmes vocaliques s'ajoutent trois diphtongues. L'ensemble constitue vingt-et-un phonèmes vocaliques. En thaï, le phonème vocalique ne peut pas se prononcer s'il n'est pas porté par un phonème consonantique.

Ces tableaux nous permettent de constater non seulement les phonèmes vocaliques qui sont communs dans ces deux langues : /a/, /i/,/e/, / $/ \mathrm{e} / \mathrm{/} / \mathrm{/} / \mathrm{u} /$ et /o/ mais aussi plusieurs spécificités vocaliques qui diffèrent manifestement. Celles présentes en thaï mais totalement inconnues des Français comprennent d'une part les phonèmes longs de chacun des précédents:/a:/, /i:/, /e:/, /ع:/, /o:/, /u:/ et /o:/ et d'autre part les phonèmes

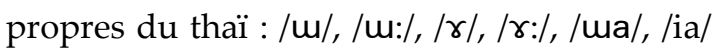
et /ua/. En revanche, le français dispose de certains phonèmes vocaliques qui n'existent

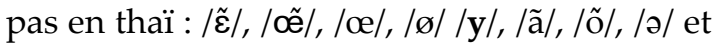
/a/.

Au-delà des différences caractéristiques concernant les consonnes et les voyelles, nous constatons une autre particularité qui oppose le thaï au français. Il s'agit des tonalités ou des variations de registres de la voix. En fait, une combinaison de consonne et voyelle n'est pas considérée comme une syllabe si elle ne porte pas de ton. Le ton joue un rôle crucial dans les langues polytonales monosyllabiques. Il est 
caractérisé d'une part par une courbe mélodique dont les composants sont importants pour identifier la perception de chaque ton et d'autre part par leur hauteur ou leur mouvement mélodique qui détermine des sens différents pour un même mot. En effet, un changement de ton dans la prononciation d'une syllabe en thaï entraîne un changement de sens du mot.

Le thaï dispose de cinq tons différents:

1. le ton "moyen » correspond à la façon naturelle de prononcer chaque syllabe ;

2. le ton «bas » correspond à une voix grave. Sa transcription phonétique est / / ;

3. le ton «descendant » va d'une voix aiguë à une voix grave dont la transcription phonétique est /^/;

4. le ton «haut» correspond à une voix aiguë dont la transcription phonétique est /'/ ;

5. le ton «montant» va d'une voix grave à une voix aiguë. Sa transcription phonétique est $/{ }^{\vee} /$.

Une fois que l'un de ces cinq tons est combiné avec un groupe de phonèmes, cela permet de former différents mots par exemple: le groupe de phonèmes $/ \mathrm{k}^{\mathrm{h} a m} /$ permet de former quatre mots avec différents tons :

[k $\left.\mathrm{k}^{\mathrm{h} a m}\right]($ ton moyen $)=$ un mot

[khâm] (ton descendant) = il fait nuit.

[k $\left.\mathrm{k}^{\mathrm{h}} \mathrm{m}\right]($ ton haut $)=$ soutenir

[kăm] (ton montant) = marrant, ridicule, drôle

Mais en français, une langue accentuelle, la notion de ton est inconnue. Une syllabe peut être composée d'une seule voyelle prononcée qui est entourée d'un nombre variable de consonnes. La suite de deux voyelles crée donc deux syllabes comme dans les mots suivants : [ja uRt] (yaourt), [ka o] (chaos), [pe i] (pays), [ma is] (maïs), etc. L'accent constitue un marqueur de fin de groupe accentuel comprenant un certain nombre de syllabes. C'est-à-dire que c'est toujours la dernière syllabe du groupe accentuel qui porte l'accent quel que soit le nombre de syllabes de celui-ci. Ce groupe forme une unité rythmique (Wioland, 1991).

L'analyse comparative des phonèmes consonantiques et vocaliques et le ton en thaï et en français nous montre qu'il existe un certain nombre de différences. Ces différences du système phonétique de ces deux langues constituent certainement des facteurs importants non seulement pour la prononciation mais aussi la compréhension orale $\mathrm{du}$ français par les étudiants thaïlandais apprenant le français langue étrangère. Ainsi, nous avons procédé à l'étude expérimentale pour étudier les problèmes de prononciation du français et les interférences phonologiques dans la prononciation du français chez les apprenants thaïlandais d'une part et les causes de ces problèmes d'autre part.

Les problèmes de prononciation chez les lycéens apprenant le FLE dans le Sud de la Thaïlande

Les résultats de la recherche nous révèlent que les problèmes phonétiques rencontrés par les étudiants thaïlandais apprenant le français sont nombreux. Dans cet article, nous présenterons seulement les problèmes de prononciation des sons [u], [y], [S], [3] et [в]. Ci-dessous sont montrés les tableaux des fautes de prononciation chez les apprenants thailandais.

Tableau 5. Les fautes de prononciation des sons [y] et $[\mathrm{u}]$

\begin{tabular}{cccccc}
\hline $\begin{array}{c}\text { mots } \\
\text { français }\end{array}$ & $\mathbf{N}$. & $\begin{array}{c}\text { nombre de bonnes } \\
\text { prononciations }\end{array}$ & pourcentage & $\begin{array}{c}\text { nombre } \\
\text { de mauvaises } \\
\text { prononciations }\end{array}$ & pourcentage \\
\hline jupe & 30 & 22 & 73.33 & 8 & 26.67 \\
brûler & 30 & 10 & 33.33 & 20 & 66.67 \\
roue & 30 & 14 & 46.67 & 16 & 53.33 \\
pour & 30 & 21 & 70 & 9 & 30 \\
vu & 30 & 24 & 80 & 6 & 20
\end{tabular}




\begin{tabular}{|c|c|c|c|c|c|}
\hline bu & 30 & 22 & 73.33 & 8 & 26.67 \\
\hline lune & 30 & 13 & 43.33 & 17 & 56.67 \\
\hline lutte & 30 & 18 & 60 & 12 & 20 \\
\hline flûte & 30 & 19 & 63.33 & 11 & 36.67 \\
\hline minute & 30 & 20 & 66.67 & 10 & 33.33 \\
\hline dessus & 30 & 2 & 6.67 & 28 & 93.33 \\
\hline russe & 30 & 12 & 40 & 18 & 60 \\
\hline rouge & 30 & 10 & 33.33 & 20 & 66.67 \\
\hline joue & 30 & 16 & 53.33 & 14 & 46.67 \\
\hline jus & 30 & 5 & 16.67 & 25 & 83.33 \\
\hline doux & 30 & 15 & 50 & 15 & 50 \\
\hline rue & 30 & 15 & 50 & 15 & 50 \\
\hline pur & 30 & 4 & 13.33 & 26 & 86.67 \\
\hline
\end{tabular}

Ce tableau nous représente que les lycéens thaïlandais ont des problèmes à différencier les sons [y] et [u]. Dans la plupart des cas, ils prononceront un $[\mathrm{u}]$ à la place d'un [y] par exemple dans les mots suivants : pur, vu, rue, jupe, lune, russe, etc. Il va de soi que lorsque le son [y] dans la langue française ne leur est pas connu, les apprenants cherchent à créer une interférence ou un transfert négatif en trouvant le son dont la prononciation est la plus proche dans leur langue maternelle (Purinthrapibal, 2010). En principe, la prononciation du son $[\mathrm{u}]$ en français n'est pas tout à fait comparable à celle des sons $[u]$ ou [u:] mais elle est très proche $\mathrm{du}[\mathrm{u}]$ en thaï. Donc, les lycéens thaiilandais se servent du [u:] présents en thaï comme dans le mot ปู [pu:] pour prononcer consécutivement le [y] dans le mot «pur » en français.

Pourtant, au-delà du recours à un certain son dans la langue thaï dont la prononciation est proche pour prononcer un mot en français, les fautes de prononciation de certains mots dans la liste ci-dessus proviennent également $d$ 'autres facteurs. Certains apprenants thaillandais ont prononcé une consonne finale dans les mots "jus" comme [3us] ou [3ys], «doux» comme [duks] et «dessus » comme [dessus], [dəsys], [dəsus] ou [desus]. Ces phénomènes seraient probablement relatifs à la complexité des systèmes phonétiques du français. D'autres ont eu une difficulté de prononcer le son [e] à la fin du verbe infinitif comme dans le mot «brûler» qui s'est prononcé comme [brylə],

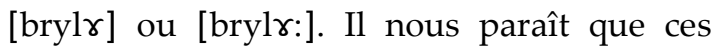
fautes sont influencées soit par la langue anglaise: en anglais le graphème /er/ se prononce en [ə] soit par la langue thaï qui se dispose de deux sons [ $\gamma]$ et [ $\gamma:]$. Dans ces cas, les apprenants thaïlandais essaient d'une part de trouver le son qui existe dans leur langue maternelle comme dans les mots thaï เฉอะ $[1 \gamma]$,

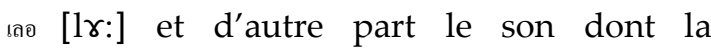
prononciation est la plus proche dans leur langue maternelle ou dans une autre langue étrangère, c'est-à-dire l'anglais, leur première langue étrangère apprise.

Tableau 6. Les fautes de prononciation des sons [3] et [S]

\begin{tabular}{lccccc}
\hline mots français & N. & $\begin{array}{c}\text { nombre de bonnes } \\
\text { prononciations }\end{array}$ & pourcentage & $\begin{array}{c}\text { nombre } \\
\text { de mauvaises } \\
\text { prononciations }\end{array}$ & pourcentage \\
\hline
\end{tabular}

\begin{tabular}{clllll}
\hline bonjour & 30 & 16 & 53.33 & 14 & 46.67 \\
\hline
\end{tabular}




\begin{tabular}{clllll}
\hline Gérard & 30 & 5 & 16.67 & 25 & 83.33 \\
chou & 30 & 20 & 66.67 & 10 & 33.33 \\
chant & 30 & 19 & 63.33 & 11 & 36.67 \\
bouche & 30 & 17 & 56.67 & 13 & 43.33 \\
manger & 30 & 17 & 56.67 & 13 & 43.33 \\
jupe & 30 & 22 & 73.33 & 8 & 26.67 \\
argent & 30 & 10 & 33.33 & 20 & 66.67 \\
Argentine & 30 & 12 & 40 & 18 & 60 \\
gens & 30 & 13 & 43.33 & 17 & 56.67 \\
joue & 30 & 16 & 53.33 & 14 & 46.67 \\
jus & 30 & 5 & 16.67 & 25 & 83.33 \\
bouge & 30 & 8 & 26.67 & 22 & 73.33 \\
\hline
\end{tabular}

Quant à la prononciation des [3] et [S] que les Thaïlandais ne connaissent pas, notre étude montre aussi que les apprenants thaïlandais du FLE se servent les mêmes stratégies que le cas précédent. Comme le thaï ne dispose pas de constructives sonores, les étudiants thaïlandais ont tendance à articuler le son qui leur est plus familier, c'est-à-dire le [S] à la place du [3] comme dans les mots «bonjour, jupe, manger, argent, Argentine, gens, bouge». En effet, la prononciation du $\left[\int\right]$ est plus proche au son
[ $\left[\epsilon^{\mathrm{h}}\right]$ et $[\mathrm{t} \varphi]$ en thaï que celle du son [3] en français. Ainsi, dans la plupart des cas, nous trouvons que les fautes des mots se composant de /ch/ comme "chou, chant, couche, bouche" ne sont pas nombreuses parce que le son [ $\left.\int\right]$ est proche du [ $\left.t c\right]$ en thaii.

Au-delà des difficultés de différencier et prononcer les sons [y] et [u] et [3] et [S], les lycéens thaïlandais ont une grande difficulté de prononcer lе [в] qui n'existe pas en thaï. Ci-dessous sont montrées les fautes de prononciation du son $[\mathrm{s}]$.

Tableau 7. Les fautes de prononciation du son [ь]

\begin{tabular}{cccccc}
\hline mots français & $\mathbf{N}$. & $\begin{array}{c}\text { nombre de bonnes } \\
\text { prononciations }\end{array}$ & pourcentage & $\begin{array}{c}\text { nombre } \\
\text { de mauvaises } \\
\text { prononciations }\end{array}$ & pourcentage \\
\hline montre & 30 & 13 & 43.33 & 17 & 56.67 \\
Gérard & 30 & 5 & 16.67 & 25 & 83.33 \\
Rien & 30 & 13 & 43.33 & 17 & 56.67 \\
Robe & 30 & 7 & 23.33 & 23 & 76.67 \\
raison & 30 & 19 & 63.33 & 11 & 36.67 \\
brûler & 30 & 10 & 33.33 & 20 & 66.67 \\
royal & 30 & 2 & 6.67 & 28 & 93.33 \\
parole & 30 & 2 & 6.67 & 28 & 93.33 \\
fenêtre & 30 & 14 & 46.67 & 16 & 53.33 \\
russe & 30 & 12 & 40 & 18 & 60 \\
\hline
\end{tabular}




\begin{tabular}{cccccc}
\hline rouge & 30 & 10 & 33.33 & 20 & 66.67 \\
riz & 30 & 12 & 40 & 18 & 60 \\
\hline
\end{tabular}

En ce qui concerne le [в], nous trouvons que dans certains cas, les élèves thaïlandais prononcent le $[r]$ ou le $[r]$ à l'anglaise à la place du [в]. En effet, l'anglais est leur première langue étrangère apprise depuis l'école primaire. Ils prennent donc l'habitude articulatoire précédemment acquise dans cette langue et réalisent le transfert négatif pour prononcer le son [в] en français.

La liste des problèmes de prononciation rencontrés par les lycéens dans le Sud de la Thaïlande ci-dessus n'est pas exhaustive. Pourtant, cette étude nous montre plusieurs constatations qui permettent d'affirmer les études précédentes menées auprès des apprenants thaïlandais du FLE dans d'autres régions. Telles sont celle de Ngammana (2011) qui ont étudié les problèmes de prononciation des élèves du français dans les écoles secondaires en Thaïlande, celle de Boonprome (2009a, 2009b) ayant étudié les problèmes de prononciation des étudiants en français à l'université de Chiangmai dans le Nord de la Thaïlande, celles de Roungtheera (2011) et Le Corré (2013) faisant les études des problèmes de prononciation des étudiants en français à l'université de Mahasarakham et à l'université de Khon Kaen dans le Nord-Est de la Thaïlande.

\section{CONCLUSION}

En conclusion, les résultats de la recherche nous mettent en évidence que les problèmes de prononciation chez les apprenants thaïlandais de FLE sont diverses. Ils nous permettent de confirmer que la langue maternelle a toujours une influence lors de l'apprentissage d'une langue étrangère. Autrement dire, les apprenants font soit une transposition d'une habitude propre au système linguistique de la langue maternelle (manière de distribuer les sons) soit une transposition propre aux habitudes phonétiques (manière de prononcer les sons). Ces causes s'influencent réciproquement et les séparer est artificiel (Léon, 1992). Au-delà, d'autres langues étrangères apprises et mieux maîtrisées par les apprenants, en particulier l'anglais, et la complexité des systèmes phonétiques $\mathrm{du}$ français sont aussi considérées comme des causes importantes des problèmes de prononciation chez ces apprenants thaïlandais. Ces événements de problèmes de prononciation constituent un grand obstacle non seulement à la production orale mais aussi à l'acquisition d'une langue étrangère notamment chez les étudiants thaillandais qui ont peu d'occasion de pratiquer la langue dans une situation réelle.

Ainsi, il va de soi que l'aspect phonétique ne doit pas être oublié lors de notre enseignement et notre organisation de l'apprentissage du FLE. Pour assister nos apprenants à corriger leurs fautes de prononciation et à progresser rapidement dans leur apprentissage du FLE, nous pouvons mettre en place différentes activités et outils comme a proposé Le Corré (2013) : un travail régulier matérialisé par des exercices d'écoute, de souplesse articulatoire visant à une meilleure fluidité et de sensibilisation au rythme et à l'accentuation des groupes syntaxiques. En effet, la phonétique permet à nos apprenants non seulement de bien prononcer en français mais de bien comprendre en français. Comme a dit Rey (2010) : "c'est grâce à leur prononciation correcte que les étudiants seront fiers de pratiquer le français en dehors de la classe. Premièrement parce que cela leur vaudra des compliments de la part des francophones et ensuite parce qu'ils n'éprouveront alors pas de honte à l'utiliser : combien de nos étudiants n'osent pas parler en classe parce qu'ils ont peur que quelqu'un ne se moque d'eux ou parce que même le professeur a du mal à les comprendre malgré un lexique et une syntaxe correcte... Et le plaisir que l'on peut ressentir à parler une langue étrangère est directement lié à notre capacité à nous faire comprendre. Si un apprenant prononce bien, cela signifie qu'il perçoit correctement chaque phonème du 
français et donc qu'il comprend mieux ce qu'on lui dit. Ceci est un atout non négligeable pour une meilleure mémorisation des structures".

\section{REMERCIEMENTS}

Je tiens à remercier la faculté des Sciences Humaines et Sociales, Université Prince de Songkla, campus de Pattani pour son soutien financier attribué à la réalisation de cette recherche.

\section{RÉFÉRENCES}

Boonprome, J. (2009a). The error system in French pronunciation by Thai learners (Rapport de recherche). Chiangmai: Chiangmai University.

Boonprome, J. (2009b). Evaluation of the effectiveness of phonetic correcting strategies for the Improvement of French pronunciation ability by Thai students. Prosodies, vocalic sounds and consonant sounds. Songklanakarin Journal of Social Sciences and Humanities, 15(6), 1037-1056.

Cuq, J.P. (2003). Dictionnaire de didactique $d u$ français langue étrangère et seconde, Paris : Clé International.

Jamet, C. (2000). Contrastivité et enseignement $d u$ français langue étrangère en France : approche anthropo-didactique (Thèse de doctorat publié). Université de Lyon 2, France.

Le Corré, Ch. (2013). Etude de la prononciation Etude de la prononciation des étudiants thaïlandais en apprentissage du français et majeure de français et majeure de français à l'université de Khon Kaen, Thaïlande
(Rapport de recherche). Khon Kaen : Université de Khon Kaen.

Léon, P. (1992). Phonétisme et prononciations du français. Paris : Nathan

Ngammana, P. (2011). Les problèmes de la prononciation $d u$ français chez les lycéens Thaïlandais (Mémoire de Master publié). Université Chulalongkorn, Thaillande.

Ninlagarn, O. (1993). Analyse prosodique de différentes attitudes émotionnelles dans une langue à ton (Thèse de doctorat publié). Université Strasbourg 2, France.

Purinthrapibal, S. (2008). Enseignement du FLE assisté par ordinateur en Thaïlande : étude de l'aide à la compréhension des relations logiques cause-conséquence (Thèse de doctorat publié). Université de Toulouse II, France.

Purinthrapibal, S. (2010). Language 1 and foreign language learning. Journal of Humanities and Social Sciences, Prince of Songkla University, 6(2), 47-77.

Rey, L. (2010). Bien prononcer pour bien apprendre: Méthodes de correction phonétique des voyelles du français pour les étudiants thaïlandais. Revue de l'association thailandaise des professeurs de français, 33(2), 7-22.

Roungtheera, Th. (2011). Investigating pronunciation problems of French Vowels by Thai learners. Songklanakarin Journal of Social Sciences and Humanities, 15(5), 857-869.

Wioland, F. (1991). Prononcer les mots $d u$ français, Paris: Hachette. 


\section{Annexe}

Test de prononciation

I. Test de la prononciation des mots.

\begin{tabular}{|c|c|}
\hline 1. Bonjour & 2. Thaillandaise \\
\hline 3. $\quad$ Petit & 4. $\quad$ Puis \\
\hline 5. Père & 6. Bleu \\
\hline 7. Mademoiselle & 8. $\quad$ Raison \\
\hline 9. $\quad$ Mer & 10. Brûler \\
\hline 11. Seize & 12. Wagon \\
\hline 13. Français & 14. Famille \\
\hline 15. Port & 16. Bord \\
\hline 17. Dos & 18. Emprunter \\
\hline 19. Doux & 20. Noël \\
\hline 21. Montre & 22. Faim \\
\hline 23. Payer & 24. Royal \\
\hline 25. Ville & 26. Trop \\
\hline 27. Garçon & 28. Champagne \\
\hline 29. Sac & 30. Tour Eiffel \\
\hline 31. Six & 32. Paix \\
\hline 33. Parfum & 34. Champs \\
\hline 35. Croissant & 36. Pomme \\
\hline 37. Deuxième & 38. Pluie \\
\hline 39. Chat & 40. Coup \\
\hline 41. Gérard & 42. Vrai \\
\hline 43. Manger & 44. Sel \\
\hline 45. Rien & 46. Parole \\
\hline 47. Jupe & 48. Sotte \\
\hline 49. Argent & 50. Juin \\
\hline 51. Eléphant & 52. Fenêtre \\
\hline 53. Patte & 54. Huit \\
\hline 55. Robe & 56. Travailler \\
\hline 57. Cheval & 58. Joint \\
\hline 59. Ecole & 60. Cantine \\
\hline 61. Américain & 62. Canadien \\
\hline 63. Brun & 64. Argentine \\
\hline 65. Enfantin & \\
\hline
\end{tabular}

II. Test de la prononciation des paires minimales.

\begin{tabular}{|llc|}
\hline 1. & vu & bu \\
\hline 2. & dix & dise \\
\hline 3. & baisse & basse \\
\hline 4. & peu & peur \\
\hline
\end{tabular}

\begin{tabular}{|c|c|}
\hline 5. cent & cinq \\
\hline 6. pâte & pas \\
\hline 7. bol & vol \\
\hline 8. chaude & chose \\
\hline 9. canne & camp \\
\hline 10. lune & lutte \\
\hline 11. fruit & flûte \\
\hline 12. signe & singe \\
\hline 13. nuit & nuire \\
\hline 14. fille & fil \\
\hline 15. minuit & minute \\
\hline 16. lent & $\operatorname{lin}$ \\
\hline 17. gant & gain \\
\hline 18. dessous & dessus \\
\hline 19. russe & rouge \\
\hline 20. peint & peigne \\
\hline 21. coussin & cousin \\
\hline 22. monde & mode \\
\hline 23. poison & poisson \\
\hline 24. habit & habile \\
\hline 25. peigne & peine \\
\hline 26. banc & bain \\
\hline 27. joue & jus \\
\hline 28. doux & doute \\
\hline 29. haute & hotte \\
\hline 30. roue & rue \\
\hline 31. marrant & marron \\
\hline 32. blond & blanc \\
\hline 33. pour & pur \\
\hline 34. font & vont \\
\hline 35. gare & car \\
\hline 36. pleut & pleure \\
\hline 37. laid & laine \\
\hline 38. bouche & bouge \\
\hline 39. glace & classe \\
\hline 40. riz & lit \\
\hline 41. sache & sage \\
\hline 42. chou & joue \\
\hline 43. chant & gens \\
\hline 44. gai & quai \\
\hline 45. main & ment \\
\hline
\end{tabular}

